\title{
Concordance of Self- and Informant-Rated Depressive Symptoms in Nursing Home Residents with Dementia: Cross-Sectional Findings
}

Julie Lorraine O'Sullivan ( $\sim$ julie.osullivan@charite.de )

Charité - University Medicine Berlin

Roxana Schweighart

University of Vechta

Sonia Lech

Charité - University Medicine Berlin

\section{Eva-Marie Kessler}

Medical School Berlin

Christina Tegeler-Nonnen

Medical School Berlin

Andrea Teti

University of Vechta

Johanna Nordheim

Charité - University Medicine Berlin

Paul Gellert

Charité - University Medicine Berlin

\section{Research Article}

Keywords: Depression screening, neuropsychiatric symptoms, dementia, proxy-rating, quality of life, geriatric mental health

Posted Date: November 30th, 2021

DOI: https://doi.org/10.21203/rs.3.rs-1086870/v1

License: (c) (1) This work is licensed under a Creative Commons Attribution 4.0 International License.

Read Full License

Version of Record: A version of this preprint was published at BMC Psychiatry on April 5th, 2022. See the published version at https://doi.org/10.1186/s12888-022-03876-5. 


\section{Abstract}

Background: Depression is highly prevalent in nursing home residents living with moderate to severe dementia. However, assessing depressive symptoms in residents with dementia can be challenging and may vary by rater perspective. We aimed to investigate the concordance of, and factors associated with self- and informant-rated depressive symptoms in nursing home residents with dementia.

Methods: Cross-sectional data was collected from N=162 nursing home residents with dementia (age: 53$100 ; 74 \%$ women). Self-ratings were assessed with the Geriatric Depression Scale, while the Subscale D of the Neuropsychiatric Inventory was used for informant-ratings. Cohen's Kappa was calculated to determine concordance of both measures and of each with antidepressant medication. Associations with sociodemographic variables, self- and informant-rated quality of life, dementia stage, neuropsychiatric symptoms, functional status and antidepressant medication were analysed with multivariate linear mixed models.

Results: Concordance between self- and informant-rated depressive symptoms was minimal (Cohen's Kappa $=.22, p=.02$ ). Self-reported depression was negatively associated with self-rated quality of life ( $\beta=-.37 ; 95 \% \mathrm{Cl}$ : -.48 to $.26, p<.001)$, informant-rated quality of life $(\beta=-.31 ; 95 \% \mathrm{Cl}:-51$ to $.10, p=.003)$ and functional status ( $\beta=-.15 ; 95 \% \mathrm{Cl}:-.27$ to $.03, p=.02$ ), whilst informant-rated depression revealed negative associations with informant-rated quality of life ( $\beta=-.32 ; 95 \% \mathrm{Cl}$ : -.54 to $.11, p=.003)$ and dementia stage $(\beta=-.27 ; 95 \% \mathrm{Cl}$ : -.50 to $.03, p=.028)$. No concordance was found with antidepressant medication.

Conclusions: In line with our expectations, low agreement and unique association patterns were found for both measures. These findings indicate that both instruments address different aspects of depression und underline the need for comprehensive approaches when it comes to detecting signs of clinically relevant depressive symptoms in dementia.

Trial registration: The trial was registered with the ISRCTN registry (Trial registration number: ISRCTN98947160)

\section{Introduction}

Although cognitive and functional decline are considered hallmarks of dementia, the vast majority of people living with dementia (PWD) also experience at least one neuropsychiatric symptom (NPS) in the course of the disease $(1,2)$ and NPS are especially prevalent in nursing home residents with dementia (3). Depression is among the most frequent NPS in dementia with reported prevalence rates ranging from 20 to $60 \%(4,5)$. However, depression often goes undiagnosed and therefore untreated in PWD living in nursing homes (6-8), even though higher prevalence of depression has been reported for nursing home residents with dementia compared to residents without dementia $(9,10)$ and to community-dwelling PWD (11). 
The relationship between dementia and depression is complex (12). Depressive symptoms occurring in later life are a known risk factor for the presence of cognitive deficits and dementia (13), however late onset of depressive symptoms can also constitute an early manifestation of dementia (14). In turn, dementia is a risk factor for depression due to psychological reaction to the cognitive and behavioral changes accompanying dementia (15). Comorbid depression in dementia is associated with a profound decrease in quality of life (16), accelerated cognitive decline (17) and increased mortality (18).

A number of suitable therapeutic options exist for treatment of depression in dementia $(19,20)$. However, accurate diagnosis of depression is essential in order to initiate appropriate interventions. It has been argued that numerous disease, clinician and system-level factors may hinder the diagnosis of depression in PWD $(21,22)$. Insufficient diagnosis of depression may also lead to inappropriate use of antidepressants, which has been reported in nursing home residents with and without dementia $(23,24)$. In this regard, Kramer et al. (25) found low concordance of depression diagnosis and prescriptions of antidepressants in PWD, similar findings have been reported by Kwak et al. (26). Moreover, Wetzels et al. (27) found a negative association of antidepressant medication with quality of life in PWD, a concept that is reportedly closely linked to depression (16).

While structured clinical interviews remain the goldstandard for diagnosis of depressive symptoms in dementia, self- and informant-rated assessments scales are usually applied for assessment of depressive symptoms in PWD under the real-world conditions of long-term care facilites (20). Although informantratings can be useful to objectify PWDs' functional and cognitive status (28), they have been criticized for their paternalistic approach (29) and potential lack of validity for assessing more subjective outcomes such as well-being $(30)$ and quality of life in PWD $(31,32)$. Regarding depressive symptoms, it remains unclear, whether and how information from these two modalities actually gives a consistent picture (33). Two studies compared self-ratings of depression using the Geriatrc Depression Scale (GDS) with informant-rating using the Neuropsychiatric Inventory (NPI) and found low agreement of both measures in community-dwelling people with mild dementia $(26,33)$. Similarly, other studies have reported low to moderate agreement between self- and informant-ratings of depression in community-dwelling PWD (34, $35)$ and PWD in nursing homes $(36,37)$. In contrast however, Arlt et al. (38) found good congruence of clinician and patient-rated depression in a cross-sectional study with mostly community-dwelling PWD.

Knowlegde is sparse about associations of self- and informant-depression ratings with other patient and context-related factors in nursing home residents with dementia. Gruber-Baldini et al. (39) investigated predictors of staff-rated depression in long-term care and found associations with disease-related factors, i.e., severe cognitive impairment, behavioral symptoms, and pain, as well as living in for-profit nursing homes. In a cross-sectional study with community-living PWD, Dawson et al. (40) found self-reported depression was predicted by physical strain and role captivity. To our knowlegde, factors associated with both self- and informant-ratings of depressive symptoms in nursing home residents with moderate to severe dementia have not been investigated in one and the same study. However, a systematic review including ten cross-sectional and three longitudinal studies on characteristics of self- and informantratings of quality of life of PWD in long-term care facilities revealed disparate association patterns within 
and across studies (16). For example, a population-based study demonstrated that self-ratings of quality of life are related to depression in PWD, whereas staff informant-ratings are more related to NPS and level of cognitive functioning (41). Similarly, a cross-sectional study by Beer et al. (32) also found that informant-ratings of quality of life are related to NPS, severe cognitive impairment and falls, whilst selfrated quality of life was related to physical restraint and pain. While there are hints about the differential association patterns between depressive symptoms and self- and informant-rated quality of life, the concordance of self- and informant-rated depressive symptom assessements in PWD and the specific association patterns with other constructs needs further investigation.

The present study

The aim of this cross-sectional study was (1) to determine the concordance between self- and informantrated depressive symptoms in nursing home residents living with dementia (2) to investigate factors associated with both modalities and (3) to assess concordance of self- and informant-rated depressive symptoms with antidepressant medication. Based on previous findings, we expect only moderate concordance between self- and informant-rated depressive symptoms. We expect self-rated depression will be associated subjective factors such as functional abilty, meaning the capacity to carry out activities of daily living independently and self-reported quality of life, whilst informant-rated depressive symptoms will be associated with with caregiver and disease-related factors such as severity of NPS, dementia stage and informant-rated quality of life. Furthermore, low concordance of depressive symptoms with antidepressant medication is expected.

\section{Materials And Methods}

\section{Participants and recruitment}

The present study took place within the scope of the research project PflegeTab (English language: CareTab). The primary objective of PflegeTab was to investigate the effects of a tablet-based intervention for nursing home residents with moderate to severe dementia in a cluster-randomized controlled trial. The main results of the trial have been published elsewhere (42). In the present paper, we report crosssectional analyses of baseline data collected in ten nursing homes located in Berlin, Germany. All participants were long-term residents of the included nursing homes. As the focus of the PflegeTab study was on nursing home residents with dementia, only residents with a pre-existing dementia diagnosis were screened for eligibility. In nine participating facilities, all residents with dementia were screened. The remaining facility had a special dementia unit, in this case only PWD from this unit were included in the further recruitment process. Inclusion criteria were dementia diagnosis or cognitive impairment meaning a Mini-Mental State Examination (MMSE) (43) score of less than 24 points. Exclusion criteria were preexisting severe mental and behavioural disorders other than depression and dementia, and short-term residency of less than four weeks. Legal guardians of eligible PWD were first contacted by telephone. Upon their written consent, PWD were approached and thoroughly informed about the study. Comprehensive verbal and written information was provided for both PWD and guardians about the 
research project and the trial. The PflegeTab study was conducted in accordance with the Declaration of Helsinki and was reviewed and approved by the Ethics Committee of the Medical University of Berlin (Charité - Universitätsmedizin Berlin; EA1/013/16) and registered with the ISRCTN registry (Trial registration number: ISRCTN98947160).

\section{Measures and Procedure}

Two trained and experienced research assistants visited each nursing home and collected self- and informant-rated data on site. Self-rated assessments were conducted as interviews directly with PWD. The research assisstants were instructed to approach all participating PWD to obtain self-reported data, regardless of their dementia stage. However, self-reports were not to be continued if PWD declined or if they seemed overwhelmed or distressed by the interview questions. Informant data on participants was assessed from members of the nursing home staff (trained nursing professionals) who had worked with the participant on a regular basis and thus knew them well.

\section{Depressive symptoms}

Self-reports of depressive symptoms were measured using the GDS-15 (44), which is a 15-item questionnaire in a yes/no format. Scores range from 0-15, higher scores indicate a higher risk of depression. Scores of 0-4 indicate no clinical depression, scores of 5-8 indicate mild depression, scores of 9-11 indicate moderate depression and scores of 12-15 indicate severe depression. Although the GDS-15 is not recommended for people with severe dementia (45), we approached all participants in an attempt to allow them to give self-ratings if possible. The Subscale D of the Neuropsychiatric Inventory - Nursing Home version (NPI-NH) (46) questionnaire was used to measure informant-rated depressive symptoms. Trained nursing professionals are first asked a screening item consisting of three questions to determine if depression is present or not: "Does the resident seem sad or depressed? Does he/she say that he/she feels sad or depressed? Does the resident cry at times?" If any screening question is answered with "yes", subquestions are asked to confirm the presence of depression. If depression is confirmed, informants are asked to rate frequency and severity of depression. Possible scores range from 0 (no sign of depression) to 12 (frequent and severe signs of depression) and are computed by multiplying severity ( $1=$ mild $3=$ severe) by frequency ( $1=$ rarely $-4=$ very often).

\section{Other study variables}

Self-rated quality of life was measured with the Quality of Life in Alzheimer's Disease (QOL-AD) questionnaire in PWD who were able to respond to the questions. Within the 13-item QOL-AD, participants are asked to rate different aspects of their lives on a 4-point Likert scale, total scores range from 13-52. Additionally, informant reports of quality of life were assessed with the QUALIDEM scale (47). QUALIDEM consists of 37 items belonging to nine subscales. All items are rated on a 4-point Likert scale, total scores range from 0-111. In accordance with previous studies, we computed a total score for the QUALIDEM scale (48). Higher scores reflect higher quality of life levels in both measures. Dementia stage was measured with the Functional Assessment Staging (FAST), which consists of 7 major functional stages and 11 substages. Stage levels increase as dementia progresses with stage 4 corresponding to mild 
dementia, stage 5 to moderate, stage 6 to moderately severe, and stage 7 to severe dementia. (49). Functional status was assessed with the Barthel Index (50). Scores range from 0-100. Higher Scores reflect higher functional ability. Neuropsychiatric symptoms were assessed with the informant-rated NPI$\mathrm{NH}$, which evaluates 12 NPS commonly observed in PWD using standardized interview questions. Nursing professionals are asked to rate the frequency and severity of each neuropsychiatric symptom. Scores are computed for each symptom by multiplying severity ( $1=$ mild $-3=$ severe) by frequency ( $1=$ rarely $-4=$ very often). Higher scores represent higher degrees of NPS. As we analysed the Subscale D separately, a composite score for neuropsychiatric symptoms was computed by adding up the scores of the 11 remaining NPI-NH subscales. We assessed the intake of antidepressant medication by examining patient records. The presence of antidepressants was coded as $0=$ not present or $1=$ present and information was taken from medical records of the facilities. The PRISCUS list, the German equivalent of the Beers list, was used to assess potentially inadequate antidepressants (51).

\section{Statistical analysis}

Descriptive statistics are reported as means and standard deviations for continuous variables, and as absolute and relative frequencies for categorical variables. Frequency of missing data was below $5 \%$ for informant-rated measures. Missing data occurred at item and scale level on all self-reported measures. Values on self-reported depressive symptoms (GDS-15) were substituted with the mean of an individual participants non-missing items if the majority of scale items $(\geq 8)$ were available. For self-reported quality of life scores (QOL-AD), a maximum of two missing items were replaced with the mean score of the remaining items, as recommended by Logsdon, Gibbons (52). Distribution of the data was not normal, therefore non-parametric Mann-Whitney U Tests were used to compare participants with and without selfratings of depressive symptoms. We calculated Spearman's correlation coefficient and Cohen's Kappa statistic to determine association and agreement between self- and informant-rated screening instruments of depressive symptoms and antidepressant medication (53). Linear mixed-effects models (LMM) fit by Restricted Maximum Likelihood Estimation were used to investigate associations of selfand informant-rated measures of depressive symptoms with other study variables. Generalized estimating equations (GEE) were used when more robust estimation methods lead to more stable models. Further variables (i.e., age, gender, self- and informant-rated quality of life, dementia stage, functional status, neuropsychiatric symptoms, antidepressant medication) were included as fixed covariates and a random intercept was added at nursing home-level to account for clustering of participants in nursing homes. All covariates were added to a multivariate model. Included variables were standardized to allow meaningful interpretation of coefficient estimates as $\beta$ coefficients. All statistical analyses were performed using IBM SPSS statistics software (IBM SPSS Statistics for Windows, Version 27.0. Armonk. NY: IBM Corp). All tests of significance were based on a $p<.05$ level and confidence interval of $95 \%$.

\section{Results}

A total of $\mathrm{N}=162$ nursing home residents with dementia were included in the study. The mean age was 85.0 years $(S D=7.1)$ and $74 \%$ were women. According to the informant-rated FAST score, 136 
participants (84\%) were classified as living with moderately severe dementia (FAST stage 6) and mean functional status score (Barthel Index) was 53.6 (SD = 26.2), indicating severe dependency. Table 1 shows an overview of participant characteristics. 
Table 1

Participant characteristics, $\mathrm{N}=162$.

\section{Characteristic}

Demographics

Age (years), M (SD)

Women, $\mathrm{n}(\%)$

Care level, n (\%)

Minor impairment

Substantial impairment

Serious impairment

Most severe impairment

Most severe impairment wto special care needs

Cognitive Status

MMSE, M (SD)

Type of Dementia, $\mathrm{n}(\%)$

Alzheimer's Disease

Vascular Dementia

Unspecified Dementia

Mixed Dementia

Others

Depressive Symptoms

Self-rated GDS-15, M (SD)

Informant-rated NPI-NH Subscale D M (SD)

Quality of Life

Self-rated QOL-AD, M (SD)

Informant-rated QUALIDEM, M (SD)

Dementia stage FAST, $\mathrm{n}(\%)$ n

162

$85.0(7.1)$

$162 \quad 119(74)$

158

$0(0)$

2 (1)

$51(32)$

$83(53)$

$22(14)$

$117 \quad 14.6(6.42)$

154

$29(19)$

$15(10)$

$77(50)$

$17(11)$

$16(10)$

$121 \quad 3.8(3.0)$

$161 \quad 1.7(2.8)$

$128 \quad 28.8(9.1)$

$161 \quad 77.4(14.3)$

161

Note $\mathrm{M}=$ Mean, $\mathrm{SD}=$ Standard Deviation, GDS-15 = Geriatric Depression Scale, NPI-NH = Neuropsychiatric Inventory - Nursing Home Version, QOL-AD = Quality of Life in Alzheimer's Disease, FAST = Functional Assessment Staging. 


\section{Characteristic}

Mild dementia

Moderately severe dementia

Severe dementia

Functional Status

Barthel Index score, M (SD)

Neuropsychiatric Symptoms

Informant-rated NPI-NH, M (SD)

Psychotropic Medication

Antidepressant present, $\mathrm{n}(\%)$
Mid-stage dementia

n

$5(3)$

$2(1)$

$136(84)$

$18(12)$

161

$53.6(26.2)$

161

$16.6(16.3)$

Note: $\mathrm{M}=$ Mean, $\mathrm{SD}=$ Standard Deviation, GDS-15 = Geriatric Depression Scale, NPI-NH = Neuropsychiatric Inventory - Nursing Home Version, QOL-AD = Quality of Life in Alzheimer's Disease, FAST = Functional Assessment Staging

Self-rated reports of depressive symptoms (GDS-15) were obtained from 121 participants. Fourty-five PWD (37\%) were classified as depressed (GDS-15 score $\geq 5), 69 \%$ of them were women. According to the GDS-15, 35 participants had mild, 8 had moderate, and 2 had severe depression. Data from 161 participants with available informant-ratings of depressive symptoms (NPI-NH subscale D) were analyzed. A total of 63 participants (39\%) were classified as depressed by the NPI-NH subscale D, $79 \%$ of them were women. There was a weak correlation between NPI-NH Subscale D and GDS-15 scores $(r=.23, p=.01)$. Dementia stage assessed with FAST was higher in participants who were not able to give self-ratings of depressive symptoms compared to those who could $(U=1575.0, p<.001)$. For the 120 PWD with available self- and informant-rated data, a Kappa coefficient of .22 ( $p=.02)$ was calculated between GDS-15 and NPI-NH subscale D, indicating a minimal level of agreement between both measures (53). Table 2 shows the classification of all participants with available data on both screening instruments.

Daily intake of antidepressants was reported for 51 (32\%) of the 162 PWD included in the study. While 41 of them were prescribed one antidepressant, 10 PWD took two antidepressants daily. No concordance of GDS-15 scores and antidepressant medication was found (Kappa coefficient $=.03 ; p=.77$ ), 36\% of those classified as depressed and $33 \%$ of those not classified as depressed with GDS- 15 received antidepressant treatment. As for classification by the NPI-NH Subscale D, $38 \%$ of those with and $28 \%$ of those without depression received antidepressants, which also indicates no agreement (Kappa coefficient=.11; $p=.16)$. Intake of a potentially inadequate drug was reported for 4 participants $(2 \%)$. 
Table 2

Prevalence and classification of depression according to GDS-15 and NPI-NH Subscale D, $n=120$

\section{Classification according to NPI-NH}

Subscale D

\begin{tabular}{|c|c|c|c|c|}
\hline & & No depression & depression & Total \\
\hline \multirow[t]{3}{*}{$\begin{array}{l}\text { Classification according to } \\
\text { GDS- } 15\end{array}$} & $\begin{array}{l}\text { No } \\
\text { depression }\end{array}$ & $53(44 \%)$ & $23(19 \%)$ & $\begin{array}{l}76 \\
(63 \%)\end{array}$ \\
\hline & Depression & $21(18 \%)$ & $23(19 \%)$ & $\begin{array}{l}44 \\
(37 \%)\end{array}$ \\
\hline & Total & $74(62 \%)$ & 46 (38\%) & 120 \\
\hline
\end{tabular}

Note: GDS-15 = Geriatric Depression Scale 15 items, NPI-NH = Neuropsychiatric Inventory - Nursing Home Version.

Separate multivariate LMM analyses were conducted for informant- and self-rated measures of depressive symptoms. Higher self-rated depressive symptoms were associated with higher self-rated quality of life $(\beta=-.37 ; 95 \% \mathrm{Cl}$ : -.48 to $-.26, p<.001)$, higher informant-rated quality of life $(\beta=-.31 ; 95 \% \mathrm{Cl}:-51$ to $-.10, p=.003)$ and lower functional status ( $\beta=-.15 ; 95 \% \mathrm{Cl}:-.27$ to $-.03, p=.02)$, see Table 3 . For informantrated depressive symptoms, multivariate analysis revealed higher informant-rated depressive symptoms were associated with higher informant-rated quality of life ( $\beta=-.32 ; 95 \% \mathrm{Cl}$ : -.53 to -.11, $p=.003)$ and lower dementia stage ( $\beta=-.27 ; 95 \% \mathrm{Cl}$ : -.50 to $-.03, p=.03$ ). Age, gender and antidepressant medication were not associated with self- or informant-rated depression, respectively. 
Table 3

Multivariate associations of study variables with self- and informant-rated depression

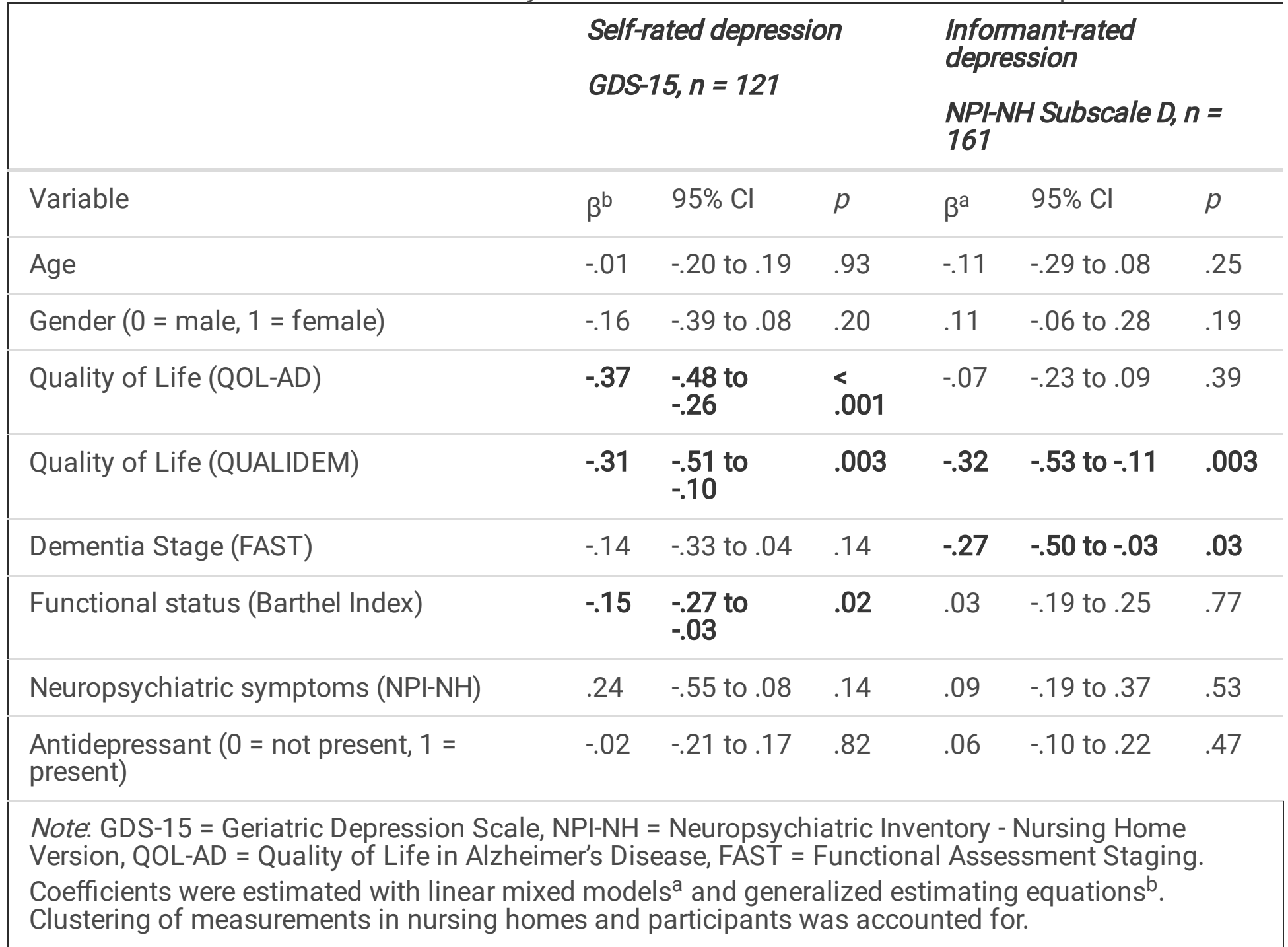

\section{Discussion}

In the present study, we investigated the agreement between self- (GDS-15) and informant-rated (NPI-NH Subscale D) depressive symptoms in nursing home residents living with dementia, factors associated with both screening measures and concordance of both modalities with antidepressant medication. In line with our expectations, we found minimal agreement between self- and informant-ratings and disparate association patterns with other study variables.

\section{Concordance of self- and informant-rated depressive symptoms}

We found high prevalence of clinically relevant depressive symptoms measured with both self- and informant-rated measures. The prevalence of clinically relevant depressive symptoms found in our study was comparable for both modalities, with a slightly higher prevalence rate for informant-rated depressive symptoms. (i.e., $37 \%$ for self-rated depressive symptoms and 39\% for informant-rated depressive 
symptoms). This is in line with prevalence rates reported in previous studies $(5,20)$. Kwak et al. $(26)$ also reported higher prevalence of non-clinically significant informant-reported depression compared to selfreported depression in PWD with mild dementia. However, as we expected, the concordance between both screening measures was only minimal.

Discrepancies in self- and informant measures of depression may stem from deficits of PWD to rate their own mood due to impaired verbal expression and potential confounding with cognitive symptoms (54). Informant-ratings are therefore frequently used to assess symptoms in PWD and are recommended to corroborate or substitute patients' self-reports (55). Research on prevalence of depression in nursing home residents with dementia usually relies on informant-reports, which are mostly obtained from clinicians or staff members such as trained nursing professionals (40). However, deviations in self- and informant-ratings can reflect biases related to negative views of informants about PWD, increased caregiver burden or misinformation about depression in dementia, going along with a systematic overestimation of depression $(34,56,57)$. Furthermore, informant-ratings can only rely on observed behavior and become increasingly biased in PWD who can not adequately communicate how they are feeling due to pain, cognitive impairment or NPS such as apathy (5). Therefore, future studies should focus on specific properties of self- and informant-ratings of depressive symptoms in PWD in order to identify and minimize inherent biases.

Not all participants in our study were able to give self-reports, and dementia stage was more advanced in those participants. In the course of dementia, progressing cognitive impairment is a known challenge. Self-reports can lead to excessive demands in people with severe dementia which makes it difficult for them to give accurate reports of their conditions. Balsamo et al. (58) recently reviewed self-report measures for assessment of depressive symptoms in older adults and concluded that many of the available screening instruments (e.g., Becks Depression Scale-II, Center for Epidemiological Studies Depression Scale, Zung Self-rating Depression Scale) do not sufficiently consider cognitive or sensory impairments among older patients. Although the GDS was specifically developed for geriatric patients and contains less somatic items than other screening measures, the validity of the GDS can be diminished in people with moderate to severe dementia $(20,59)$. Therefore, informant-rated screening tools such as the Subscale D of the NPI may offer a pragmatic approach for widespread depression diagnosis in everyday nursing home settings (60). Nonetheless, whenever possible, it is important to ascertain the views of PWD, as there can be large discrepancies between staff and PWD depression ratings (61). PWD differ in their level of insight and deterioration of a sense of self (62). Self-reports strengthen and support autonomy in PWD and allow them to express their own views $(29,63)$. Snow et al. (64) found that an existing diagnosis of dementia per se does not lead to inaccurate self-reports of depression. Perfect et al. (65) investiged challenges and benefts of using self-reports in research with PWD. They conclude that self-reports can and should be applied in studies with people in all stages of dementia. Our findings underscore the importance of incorporating self-reports when assessing depressive symptoms in PWD, as some internalized depressive symptoms such as feelings of worthlessness, guilt and loneliness may not be evident to external informants $(33,40)$. 


\section{Factors associated with both self- versus informant-rated depressive symptoms}

Both measures showed divergent association patterns with other study variables. In line with our expectations, higher self-rated depressive symptoms were associated with lower self-rated quality of life and lower fuctional abilty, whereas higher informant-rated depressive symptoms were associated with lower informant-rated quality of life. Our results confirm the widely reported link between depression and quality of life in PWD (32). We observed a method factor for both modalities showing associations of self- and informant-measures of depressive symptoms and quality of life, respectively. There was also an unexpected crossover from self-reported depressive symptoms to informant-reported quality of life: Higher self-reported depressive symptoms was related to lower informant-rated quality of life, but not vice versa.

In line with previous findings $(66,67)$, we found depressive symptoms to be lower in participants with higher dementia severity. However, this was only true for informant-rated depression. In contrast, selfrated depression was related to lower functional status. Knapskog, Barca (68) found impaired ADL was clearly associated with depression in PWD, and Crespo, Hornillos (69) found functional autonomy to be a main predictor of informant-rated quality of life in nursing home residents with dementia. Taken together, our results suggest that depression may go underrecognized by staff informants in residents with higher stages of dementia and greater levels of functional impairment, as their focus may shift to other symptoms (11). Furthermore, staff informants may not always recognize depression in residents with severe dementia due to the overlap of depression and dementia symptoms $(59,70)$. This could lead to an undersupply of therapeutic interventions in PWD with moderate to severe dementia. It has been recommended that intervention strategies should be applied in all stages of dementia, as the prevalence of depressive symptoms does not seem to be linked to the severity of dementia (60) and there is sufficient evidence showing that depression in dementia is treatable $(19,20)$. Nursing home staff should receive extensive training on detection of depression in dementia, in order to distinguish between overlapping symptoms of depression and dementia (35). Taken together, the unique association patterns of self- and informant-rated depression with other variables indicate that depression has multidimensional aspects, underlining the importance of multimodal approaches in order to reach an accurate and comprehensive assessment of depressive symptoms in PWD (34).

\section{Concordance of self- and informant-rated depressive symptoms with antidepressant medication}

In line with Kwak et al. (26), we found no significant agreement of either screening instrument with antidepressant medication. Descriptive review of antidepressant pharmacotherapy showed occasional use of potentially inadequate drugs in some participants. Although there is a growing body of research indicating that pharmacological therapy of depression in PWD is essentially ineffective and potentially harmful (71), it remains unclear if these empirical findings are known and considered in real world medical settings. In line with findings reported by Kramer et al. (25), our data show that a large proportion 
of participants received antidepressants, even though there were no apparent self- or informant-rated depressive symptoms. Occasional inappropriate use of antidepressants was also identified. Although antidepressants such as selective serotonin reuptake inhibitors (SSRIs) are frequently prescribed for managing depressive symptoms in dementia (72), two recent systematic reviews of pharmacological treatment of depression have reported inconclusive evidence on the efficacy of antidepressants in PWD $(71,73)$. Furthermore, there is a consensus among evidence-based treatment guidelines for dementia that pharmacological options should only be considered if non-pharmacological options have failed, in order to avoid unwanted side-effects (19). Due to the cross-sectional nature of our data, we cannot rule out that the medication was initially prescribed due to a pre-existing depression disorder. However, in light of the mixed findings on pharmacological treatment of depression, medication should be evaluated in a timely fashion and if possible reduced upon remission.

\section{Limitations}

Although our study has many strengths, some important limitations must be mentioned. Our main objective was to investigate the concordance and association patterns of self- and informant-rated depressive symptoms in nursing home residents in a cross-sectional design. Future studies should include longitudinal and factorial designs in order to shed more light on specific properties of self- and informant-ratings. A further limitation is the absence of a structured clinical interview as gold standard method for diagnosis of depression. Both instruments used in our study (GDS-15 and NPI-NH Subscale D) were designed for depression screening and can in no way substitute a full diagnostic workup conducted by trained medical experts such as clinical psychologists or physicians. Future studies should take a more comprehensive diagnostic approach to address discrepancies between self- and informant assessments and clarify whether they are systematic in nature and due to the fact that different facets of depression are being assessed, or whether they are in fact unsystematic and due to the limited reliability and validity of the measurement instruments. Innovative measurements such as Ecological Momentary Assessments may also provide further insights into fluctuating affective states, which have been reported in PWD. Finally, the GDS-15 is not recommended for patients with mild-moderate dementia, other instruments such as the Cornell Scale for Depression in Dementia (CSDD) which combines self- and clinician-ratings (74) may be more suited for patients with more severe dementia and MMSE-scores below 15. Moreover, we used two different instruments to measure agreement of informant- and selfrated depression. Future studies should utilize informant- and self-rating versions of the same instrument, in order to rule out instrument bias as compared to type-of-rating bias, which would provide valuable insights for clinical practice in nursing homes.

\section{Conclusion}

In light of high prevalence rates, depression diagnosis should be conducted routinely in nursing home residents with dementia, ideally by trained experts, such as clinical psychologists or physicians. Comprehensive screening for depressive symptoms should incorporate both self- and informant-reports whenever possible, and clinicians should be aware of the differences between self- and informant-ratings 
of depressive symptoms in PWD. While informant-ratings may be feasible is all dementia stages, they can only be used to assess visible symptoms displayed on the behavioral level. Insights about inner states, such as feelings of worthlessness and guilt need to be derived from self-reports. Therefore, self-reports are an essential part of depression diagnosis and an effort should be made to obtain self-reports from PWD regardless of their dementia stage. Adequate treatment should be offered to those affected by depression in a timely fashion, regardless of dementia severity and regular evaluation of prescribed antidepressants should be conducted, in order to avoid potential inappropriate use of antidepressants in nursing home residents with dementia.

\section{Abbreviations}

$95 \% \mathrm{Cl}$

95\% confidence interval

AES-I

Apathy Evaluation Scale - Informant Version

cRCT

cluster-randomised controlled trial

FAST

Functional Assessment Staging

GDS

Geriatric Depression Scale

GEE

Generalized Estimating Equations

LMM

linear mixed models

$\mathrm{M}$

mean

MMSE

Mini Mental State Examination

$\mathrm{NPI}-\mathrm{NH}$

Neuropsychiatric Inventory - Nursing Home Version

NPS

Neuropsychiatric symptoms

PWD

people with dementia

QOL-AD

Quality of Life in Alzheimer's Disease

REML

restricted maximum likelihood estimation

SD 
standard deviation

SSRIS

selective serotonin reuptake inhibitors

\section{Declarations}

\section{Ethics approval and consent to participate}

The PflegeTab study was conducted in accordance with the Declaration of Helsinki and was reviewed and approved by the Ethics Committee of the Medical University of Berlin (Charité - Universitätsmedizin Berlin; EA1/013/16) and registered with the ISRCTN registry (Trial registration number: ISRCTN98947160).

This study was approved by the local ethics committee of the Charité Medical University of Berlin (number EA1/013/16). Written informed consent was obtained from participants or legal guardians or prior to data collection.

\section{Consent for publication}

Not applicable.

\section{Availability of data and materials}

The datasets used and analysed are stored in a non-publicly available repository and are available from the corresponding author on reasonable request.

\section{Competing interests}

The authors declare that they have no competing interests.

\section{Funding}

This research was funded by the German National Association of Statutory Health Insurance Funds (GKVSpitzenverband Grant Number 0001). There is no financial relationship between the authors and the sponsors. The German National Association of Statutory Health Insurance Funds played no role in the design, collection, analysis, and interpretation of data, in the writing of the manuscript, or in the decision to submit the manuscript for publication. We acknowledge support from the German Research Foundation (DFG) and the Open Access Publication Funds of Charité - Universitätsmedizin Berlin. 


\section{Authors' contributions}

JLOS was the main contributor in writing the manuscript, analysing and interpreting the data. JLOS, RS, PG, and AT designed the study. JLOS, RS and SL conducted a review of the literature. SL, AT, CTN, EMK and JN made contributions to the manuscript. JLOS, RS and PG analysed the data, and all authors were involved in reviewing and interpreting the findings. All authors read and approved the final version of the manuscript.

\section{Acknowledgements}

The authors extend their special appreciation to Adelheid Kuhlmey (Charité - Universitätsmedizin Berlin) and Sebastian Möller and Jan-Niklas Voigt-Antons (Technische Universität Berlin) who designed the PflegeTab trial, in which the present study was embedded. We also thank Laura Jordan and Sophie Guinet for their efforts in collecting the data. We would also like to acknowledge Jacqueline Wienholtz, Marco Reichert, and Ines Jesse for their support in preparing and organizing the study.

\section{Authors' information}

${ }^{1}$ Charité - Universitätsmedizin Berlin, corporate member of Freie Universität Berlin, Humboldt-Universität zu Berlin, and Berlin Institute of Health, Institute for Medical Sociology and Rehabilitation Science, Charitéplatz 1, 10117 Berlin, Germany

${ }^{2}$ Institute for Gerontology, University Vechta, Driverstraße 2, D-49377 Vechta, Germany

${ }^{3}$ MSB Medical School Berlin, Department of Psychology, Rüdesheimer Str. 50, 14197 Berlin, Germany

\section{References}

1. Steinberg M, Tschanz JT, Corcoran C, Steffens DC, Norton MC, Lyketsos CG, et al. The persistence of neuropsychiatric symptoms in dementia: the Cache County Study. Int J Geriatr Psychiatry. 2004;19(1):19-26.

2. Zhao Q-F, Tan L, Wang H-F, Jiang T, Tan M-S, Tan L, et al. The prevalence of neuropsychiatric symptoms in Alzheimer's disease: Systematic review and meta-analysis. J Affect Disord. 2016;190:264-71.

3. Helvik A-S, Engedal K, Wu B, Benth JŠ, Corazzini K, Røen I, et al. Severity of Neuropsychiatric Symptoms in Nursing Home Residents. Dement Geriatr Cogn Dis Extra. 2016;6(1):28-42.

4. Nowrangi MA, Lyketsos CG, Rosenberg PB. Principles and management of neuropsychiatric symptoms in Alzheimer's dementia. Alzheimers Res Ther. 2015;7(1):12. 
5. Leung DKY, Chan WC, Spector A, Wong GHY. Prevalence of depression, anxiety, and apathy symptoms across dementia stages: A systematic review and meta-analysis. Int J Geriatr Psychiatry. 2021;36(9):1330-44.

6. Llewellyn-Jones RH, Snowdon J. Depression in nursing homes: ensuring adequate treatment. CNS Drugs. 2007;21(8):627-40.

7. Volicer L, Frijters DHM, van der Steen JT. Underdiagnosis and undertreatment of depression in nursing home residents. European Geriatric Medicine. 2011;2(6):332-7.

8. Watson LC, Garrett JM, Sloane PD, Gruber-Baldini AL, Zimmerman S. Depression in Assisted Living: Results From a Four-State Study. The American Journal of Geriatric Psychiatry. 2003;11(5):534-42.

9. Davison TE, McCabe MP, Mellor D, Ski C, George K, Moore KA. The prevalence and recognition of major depression among low-level aged care residents with and without cognitive impairment. Aging Ment Health. 2007;11(1):82-8.

10. Jongenelis $K$, Pot AM, Eisses AM, Beekman AT, Kluiter H, Ribbe MW. Prevalence and risk indicators of depression in elderly nursing home patients: the AGED study. J Affect Disord. 2004;83(2-3):135-42.

11. Bergdahl E, Allard P, Gustafson Y. Depression among the very old with dementia. International Psychogeriatrics. 2011;23(5):756-63.

12. Peakman G, Karunatilake N, Seynaeve M, Perera G, Aarsland D, Stewart R, et al. Clinical factors associated with progression to dementia in people with late-life depression: a cohort study of patients in secondary care. BMJ Open. 2020;10(5):e035147.

13. Livingston G, Huntley J, Sommerlad A, Ames D, Ballard C, Banerjee S, et al. Dementia prevention, intervention, and care: 2020 report of the Lancet Commission. The Lancet. 2020;396(10248):413-46.

14. Cooper C, Sommerlad A, Lyketsos CG, Livingston G. Modifiable predictors of dementia in mild cognitive impairment: a systematic review and meta-analysis. Am J Psychiatry. 2015;172(4):323-34.

15. Fiske A, Wetherell JL, Gatz M. Depression in older adults. Annu Rev Clin Psychol. 2009;5:363-89.

16. Beerens HC, Zwakhalen SM, Verbeek H, Ruwaard D, Hamers JP. Factors associated with quality of life of people with dementia in long-term care facilities: a systematic review. Int J Nurs Stud. 2013;50(9):1259-70.

17. Rapp MA, Schnaider-Beeri M, Wysocki M, Guerrero-Berroa E, Grossman HT, Heinz A, et al. Cognitive Decline in Patients With Dementia as a Function of Depression. The American Journal of Geriatric Psychiatry. 2011;19(4):357-63.

18. Peters ME, Schwartz S, Han D, Rabins PV, Steinberg M, Tschanz JT, et al. Neuropsychiatric symptoms as predictors of progression to severe Alzheimer's dementia and death: the Cache County Dementia Progression Study. Am J Psychiatry. 2015;172(5):460-5.

19. Ngo J, Holroyd-Leduc JM. Systematic review of recent dementia practice guidelines. Age Ageing. 2015;44(1):25-33.

20. Burke, Goldfarb, Bollam, Khokher. Diagnosing and Treating Depression in Patients with Alzheimer's Disease. Neurol Ther. 2019;8(2):325-50. 
21. Curran EM, Loi S. Depression and dementia. Med J Aust. 2013;199(S6):S40-4.

22. Agüera-Ortiz L, García-Ramos R, Grandas Pérez FJ, López-Álvarez J, Montes Rodríguez JM, Olazarán Rodríguez FJ, et al. Depression in Alzheimer's Disease: A Delphi Consensus on Etiology, Risk Factors, and Clinical Management. Frontiers in Psychiatry. 2021;12(141).

23. Hanlon JT, Wang X, Castle NG, Stone RA, Handler SM, Semla TP, et al. Potential Underuse, Overuse, and Inappropriate Use of Antidepressants in Older Veteran Nursing Home Residents. Journal of the American Geriatrics Society. 2011;59(8):1412-20.

24. Shah S, Schoenbachler B, Streim J, Meeks S. Antidepressant prescribing patterns in the nursing home: second-generation issues revisited. J Am Med Dir Assoc. 2012;13(4):406.e13-8.

25. Kramer D, Allgaier A-K, Fejtkova S, Mergl R, Hegerl U. Depression in Nursing Homes: Prevalence, Recognition, and Treatment. The International Journal of Psychiatry in Medicine. 2009;39(4):345-58.

26. Kwak YT, Yang Y, Pyo SJ, Koo MS. Clinical characteristics according to depression screening tools in patients with Alzheimer's disease: view from self, caregiver-reported and drug-intervention pattern. Geriatr Gerontol Int. 2014;14(3):660-6.

27. Wetzels RB, Zuidema SU, de Jonghe JF, Verhey FR, Koopmans RT. Determinants of quality of life in nursing home residents with dementia. Dement Geriatr Cogn Disord. 2010;29(3):189-97.

28. Taylor-Rowan M, Nafisi S, Patel A, Burton JK, Quinn TJ. Informant-based screening tools for diagnosis of dementia, an overview of systematic reviews of test accuracy studies protocol. Systematic Reviews. 2020;9(1):271.

29. Simmons SF, Schnelle JF, Uman GC, Kulvicki AD, Lee K-OH, Ouslander JG. Selecting Nursing Home Residents for Satisfaction Surveys1. The Gerontologist. 1997;37(4):543-50.

30. Kolanowski A, Hoffman L, Hofer SM. Concordance of Self-Report and Informant Assessment of Emotional Well-Being in Nursing Home Residents With Dementia. The Journals of Gerontology: Series B. 2007;62(1):P20-P7.

31. Römhild J, Fleischer S, Meyer G, Stephan A, Zwakhalen S, Leino-Kilpi H, et al. Inter-rater agreement of the Quality of Life-Alzheimer's Disease (QoL-AD) self-rating and proxy rating scale: secondary analysis of RightTimePlaceCare data. Health Qual Life Outcomes. 2018;16(1):131.

32. Beer C, Flicker L, Horner B, Bretland N, Scherer S, Lautenschlager NT, et al. Factors Associated with Self and Informant Ratings of the Quality of Life of People with Dementia Living in Care Facilities: A Cross Sectional Study. PLOS ONE. 2010;5(12):e15621.

33. Saari TT, Hallikainen I, Hintsa T, Koivisto AM. Network structures and temporal stability of self- and informant-rated affective symptoms in Alzheimer's disease. J Affect Disord. 2020;276:1084-92.

34. Leung WY, Bhar S, Reilly A, Pedell S. Discrepancies Between Community-Dwelling Individuals with Dementia and Their Proxies in Completing the Cornell Scale for Depression in Dementia: A Secondary Data Analysis. Clin Interv Aging. 2021;16:281-9.

35. Gold D, Rosowsky E, Piryatinsky I, Sinclair SJ. Comparing patient and informant ratings of depressive symptoms in various stages of Alzheimer's disease. Neuropsychology. 2020;34(5):535-50. 
36. Wongpakaran N, Wongpakaran T, van Reekum R. Discrepancies in Cornell Scale for Depression in Dementia (CSDD) items between residents and caregivers, and the CSDD's factor structure. Clin Interv Aging. 2013;8:641-8.

37. Assessment of depression in patients with Alzheimer's disease: Concordance among informants [press release]. US: American Psychological Association1991.

38. Arlt S, Hornung J, Eichenlaub M, Jahn H, Bullinger M, Petersen C. The patient with dementia, the caregiver and the doctor: cognition, depression and quality of life from three perspectives. Int $J$ Geriatr Psychiatry. 2008;23(6):604-10.

39. Gruber-Baldini AL, Zimmerman S, Boustani M, Watson LC, Williams CS, Reed PS. Characteristics Associated With Depression in Long-Term Care Residents With Dementia. The Gerontologist. 2005;45(suppl_1):50-5.

40. Dawson NT, Powers SM, Krestar M, Yarry SJ, Judge KS. Predictors of Self-Reported Psychosocial Outcomes in Individuals With Dementia. The Gerontologist. 2012;53(5):748-59.

41. Buckley T, Fauth EB, Morrison A, Tschanz J, Rabins PV, Piercy KW, et al. Predictors of quality of life ratings for persons with dementia simultaneously reported by patients and their caregivers: the Cache County (Utah) Study. Int Psychogeriatr. 2012;24(7):1094-102.

42. O'Sullivan JL, Lech S, Gellert P, Grittner U, Voigt-Antons JN, Möller S, et al. A tablet-based intervention for activating nursing home residents with dementia: results from a cluster-randomized controlled trial. Int Psychogeriatr. 2021:1-13.

43. Folstein MF, Folstein SE, McHugh PR. "Mini-mental state". A practical method for grading the cognitive state of patients for the clinician. J Psychiatr Res. 1975;12(3):189-98.

44. Yesavage JA, Sheikh JI. 9/Geriatric Depression Scale (GDS). Clinical Gerontologist. 1986;5(1-2):16573.

45. Conradsson M, Rosendahl E, Littbrand H, Gustafson Y, Olofsson B, Lövheim H. Usefulness of the Geriatric Depression Scale 15-item version among very old people with and without cognitive impairment. Aging Ment Health. 2013;17(5):638-45.

46. Cummings JL, Mega M, Gray K, Rosenberg-Thompson S, Carusi DA, Gornbein J. The Neuropsychiatric Inventory. Neurology. 1994;44(12):2308.

47. Ettema TP, Dröes R-M, de Lange J, Mellenbergh GJ, Ribbe MW. QUALIDEM: development and evaluation of a dementia specific quality of life instrument--validation. International Journal of Geriatric Psychiatry. 2006;22(5):424-30.

48. Appelhof B, Bakker C, Van Duinen-van den ljssel JCL, Zwijsen SA, Smalbrugge M, Verhey FRJ, et al. The Determinants of Quality of Life of Nursing Home Residents with Young-Onset Dementia and the Differences between Dementia Subtypes. Dementia and Geriatric Cognitive Disorders. 2017;43(56):320-9.

49. Sclan SG, Reisberg B. Functional Assessment Staging (FAST) in Alzheimer's Disease: Reliability, Validity, and Ordinality. International Psychogeriatrics. 1992;4(3):55-69. 
50. Mahoney FI, Barthel DW. Functional evaluation: The Barthel Index: A simple index of independence useful in scoring improvement in the rehabilitation of the chronically ill. Maryland State Medical Journal. 1965;14:61-5.

51. Zimmermann T, Kaduszkiewicz H, van den Bussche H, Schön G, Brettschneider C, König HH, et al. Potenziell inadäquate Medikamente bei älteren hausärztlich versorgten Patientinnen und Patienten. Bundesgesundheitsblatt - Gesundheitsforschung - Gesundheitsschutz. 2013;56(7):941-9.

52. Logsdon RG, Gibbons LE, McCurry SM, Teri L. Assessing quality of life in older adults with cognitive impairment. Psychosom Med. 2002;64(3):510-9.

53. McHugh ML. Interrater reliability: the kappa statistic. Biochem Med (Zagreb). 2012;22(3):276-82.

54. Leontjevas R, Gerritsen DL, Vernooij-Dassen MJ, Smalbrugge M, Koopmans RT. Comparative validation of proxy-based Montgomery-Åsberg depression rating scale and cornell scale for depression in dementia in nursing home residents with dementia. Am J Geriatr Psychiatry. 2012;20(11):985-93.

55. Sutin AR, Stephan Y, Terracciano A. Self-Reported Personality Traits and Informant-Rated Cognition: A 10-Year Prospective Study. J Alzheimers Dis. 2019;72(1):181-90.

56. Chang Y-P, Edwards DF, Lach HW. The Collateral Source version of the Geriatric Depression Scale: evaluation of psychometric properties and discrepancy between collateral sources and patients with dementia in reporting depression. International Psychogeriatrics. 2011;23(6):961-8.

57. Teresi J, Abrams R, Holmes D, Ramirez M, Eimicke J. Prevalence of depression and depression recognition in nursing homes. Soc Psychiatry Psychiatr Epidemiol. 2001;36(12):613-20.

58. Balsamo M, Cataldi F, Carlucci L, Padulo C, Fairfield B. Assessment of late-life depression via selfreport measures: a review. Clin Interv Aging. 2018;13:2021-44.

59. Goodarzi ZS, Mele BS, Roberts DJ, Holroyd-Leduc J. Depression Case Finding in Individuals with Dementia: A Systematic Review and Meta-Analysis. Journal of the American Geriatrics Society. 2017;65(5):937-48.

60. Verkaik R, Nuyen J, Schellevis F, Francke A. The relationship between severity of Alzheimer's disease and prevalence of comorbid depressive symptoms and depression: a systematic review. Int J Geriatr Psychiatry. 2007;22(11):1063-86.

61. Towsley G, Neradilek MB, Snow AL, Ersek M. Evaluating the Cornell Scale for Depression in Dementia as a proxy measure in nursing home residents with and without dementia. Aging Ment Health. 2012;16(7):892-901.

62. D'cruz MM. Does Alice Live Here Anymore? Autonomy and Identity in Persons Living and Dying With Dementia. Frontiers in Psychiatry. 2021;12(1194).

63. Prince, Prina, Guerchet. World Alzheimer Report 2013. London: Alzheimer's Disease International; 2013.

64. Snow AL, Kunik ME, Molinari VA, Orengo CA, Doody R, Graham DP, et al. Accuracy of self-reported depression in persons with dementia. J Am Geriatr Soc. 2005;53(3):389-96. 
65. Perfect D, Griffiths AW, Vasconcelos Da Silva M, Lemos Dekker N, McDermid J, Surr CA. Collecting self-report research data with people with dementia within care home clinical trials: Benefits, challenges and best practice. Dementia (London). 2021;20(1):148-60.

66. Holtzer R, Scarmeas N, Wegesin DJ, Albert M, Brandt J, Dubois B, et al. Depressive symptoms in Alzheimer's disease: natural course and temporal relation to function and cognitive status. J Am Geriatr Soc. 2005;53(12):2083-9.

67. Starkstein SE, Jorge R, Mizrahi R, Robinson RG. The construct of minor and major depression in Alzheimer's disease. Am J Psychiatry. 2005;162(11):2086-93.

68. Knapskog A-B, Barca ML, Engedal K. Prevalence of depression among memory clinic patients as measured by the Cornell Scale of Depression in Dementia. Aging \& Mental Health. 2014;18(5):57987.

69. Crespo M, Hornillos C, de Quirós MB. Factors associated with quality of life in dementia patients in long-term care. International Psychogeriatrics. 2013;25(4):577.

70. Downing LJ, Caprio TV, Lyness JM. Geriatric Psychiatry Review: Differential Diagnosis and Treatment of the 3 D's - Delirium, Dementia, and Depression. Current Psychiatry Reports. 2013;15(6):365.

71. Dudas R, Malouf R, McCleery J, Dening T. Antidepressants for treating depression in dementia. Cochrane Database Syst Rev. 2018;8(8):CD003944.

72. Lozupone M, La Montagna M, D'Urso F, Piccininni C, Rinaldi A, Beghi M, et al. The Challenge of Antidepressant Therapeutics in Alzheimer's Disease. Adv Exp Med Biol. 2020;1260:267-81.

73. Orgeta V, Tabet N, Nilforooshan R, Howard R. Efficacy of Antidepressants for Depression in Alzheimer's Disease: Systematic Review and Meta-Analysis. Journal of Alzheimer's Disease. 2017;58:725-33.

74. Alexopoulos GS, Abrams RC, Young RC, Shamoian CA. Cornell Scale for Depression in Dementia. Biol Psychiatry. 1988;23(3):271-84. 